\title{
El modelo de lago como técnica de interpretación forestal
}

\author{
Carlos A. Gracia
}

Departamento de Ecologia. Facultad de Biologia. Universidad de Barcelona.

\begin{abstract}
SUMMARY
LAKI:S AS COMPR IIHINSIVI: MODI:LS OF FORI:ST COMMUNITIISS

The vertical patterns of some forest parameters which are closely related to the mechanisms of light interception, such as leaf area index and chlorophyll content in leaves, are analysed in two forests of different structure; an cvergreen oak forest and a beccl forest. The regularities observed comparing the results of both forests enable us to outline some ideas aboutl the structure of the canopy.

The similarity between some aspects of the vertical organization of terrestrial and ayuatic ecosystems is shown clearly. It is suggested that the adaptative response of both types of ecosystens to the collection of energy by the primary producers probably follows similar pathways.
\end{abstract}

\section{INTRODUCCION}

La visión funcional del bosque dista muclo de haber alcanzado el grado de sintesis y madure\% de que goza el conocimiento del ccosistema lacustre. Dos razones fundamentales explican cste hecho. Por una parte, è lago es un ambiente que resulta casi cerrado al ciclo de materia mientras que el bosque recibc aportaciones y cxporta en un continuo trasiego de ésta. A escala temporal fenómenos que se producen en el lago en el período de un año, como la sucesión, requieren decenas o centenares de anos en el bosque, con lo que las dificultades de observación se hacen patentes.
Del análisis integrado de los ecosistenas terrestres resalta la necesidad de considerar una unidad funcional que en nuestro caso comesponde a la cuenca. La cuenca como unidad que se repite en el espacio, engloba en si misma una buena parte de las varia. ciones debidas a cambios de pendiente, altitud, orientación, naturaleza del suclo y catcnas de vegetación. En las cuencas el ccosistema se organiza y estructura de un modo complejo que apenas conocemos.

Los principios generales que rigen la organización de los lagos son válidos, a una cierta escala, en la organización de los ecosistemas forestales como la organización vertical del bosque y el lago. Es posible que 
a esta situación se haya llegado por convergencia como respuesta a problemas tan básicos como la captación de energía por los productores primarios o la circulación vertical de los nutrientes.

\section{DISCUSION}

\section{ENERGIA FISICA RECIBIDA}

El mecanismo fundamental de circulación de los nutrientes en el lago es la turbulencia generada por la paulatina degradación de energía que el propio lago recibe bajo la forma de vientos o de radiación. La producción está estrechamente relacionada con la turbulencia generada por esta energia, hasta el punto de que las zonas más productivas son las áreas de afloramiento del mar, o de los grandes lagos donde aquélla es máxima. Los organismos que se ven favorecidos en estas condiciones son los de vida corta y elevada tasa de reproducción, de los que son un buen ejemplo las diatomeas. En aguas con escasa turbulencia predominan organismos que, como los dinoflagelados, disponen de mecanismos capaces de generar movimientos que rompan los gradientes de nutrientes que se pueden formar en las proximidades de sus envueltas celulares. (MARGALEF, 1981).

Esta energía física degradada sobre la que descansa la producción en el ecosistema acuático, tiene su equivalente en ticrra. El viento y la radiación ponen en juego mecanismos de evapotranspiración que son tan necesarios para el movimiento ascensional de los nutrientes como lo eran los afloramientos o la mezcla vertical en los ecosiste mas acuáticos.

Puede establecerse una estrecha relación entre la producción vegetal terrestre y la cantidad de energia invertida en cvapotranspiración. Naturalmente hablamos aqui de la evapotranspiración real y no de la potencial que se ve limitada en muchas ocasiones por la falta de disponibilidad de agua. Esta relación es tan estrecha que muchos de los modelos de estimación de la producción primaria terrestre (LIETH \& WHITTAKER, 1975) utilizan como estimador de base la evapotranspiración. También en este caso, en las zonas de mayor evapotranspiración se ven favorecidas las especies de rápido desarrollo, más estrategas de la $r$ que de la $\mathrm{K}$, frente a las de crecimiento lento que se ven favorecidas en ambientes con menor evapotranspiración. Baste pensar, como ejemplo, en la rapide $z$ de crecimiento de un bosque de Eucaliptus frente a la lentitud del bosque mediterráneo.

\section{TRANSPORTE VERTICAL DE NUTRIENTES}

Estos dos fenómenos que acabamos de comentar, la turbulencia en los ecosistemas acuáticos y la evapotranspiración en los terrestres, generan mecanismos ascensionales de los nutrientes que tienden, por efecto de la gravedad, a acumularse en el sedimento $y$ en el suelo respectivamente. La ascensión en los vegetales terrestres se hace por caminos bien definidos: Ios vasos conductores, que se localizan en el interior de las estructuras de la propia plantá.

La madera con una tasa de renovación de decenas o cientos de años, no juega el mismo papel que, por ejemplo, las hojas que se renuevan anualmente en los caducifolios o cada 3.5 años en los perennifolios $y$ en las conife ras de nuestras latitudes.

De la producción neta anual de nuestros bosques las dos terceras partes se invierten en madera que aumenta asi la estructura del bosque, y sólo un tercio se contabiliza como producción de hojas y otros órganos fotosintetizadores. La madera es, por lo tanto, más un elemento de consumo que de producción $y$, si lo consideramos de este modo, se reduce la diferencia más importante que existe en la organización de los ecosistemas acuáticos y terrestres, a saber, la proporción entre la biomasa de los elementos productores y la de los consumidores, que es baja en el agua y más elevada en tierra. 


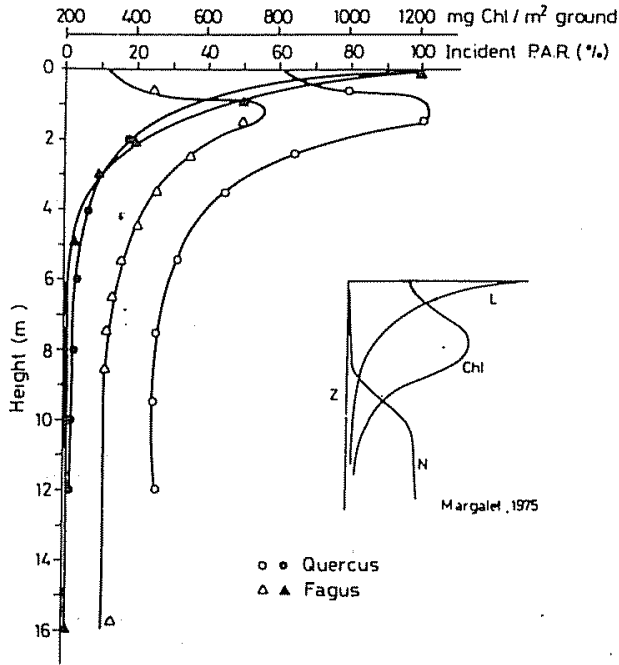

Figura 1. Distribución vertical de la clorofila total y de la radiación fotosintéticamente activa en un encinar y un hayedo del Montseny (Barcelona). Como comparación se incluye el modelo general para los sistemas acuáticos propuesto por MARGALEF (1975)

\section{RADIACION INCIDENTE Y DISTRIBUCION DE LA CLOROFILA}

El analisis comparado de la distribución de la clorofila en un encinar y en un hayedo del Montseny pone de manifiesto una estructura vertical que, en lo fundamental, es común a ambos bosques. Una característica de esta distribución es el máximo subsuper. ficial de clorofila que divide el conjunto'de la bóveda forestal en dos estratos. De una parte la capa superficial de un metro de cspesor (94 cm. en el encinar y 113 en el hayedo en el mes de agosto) y de otra la capa inferior que se extiende prácticamente hasta el suelo (fig. 1).

Casi la mitad de la superficie foliar total del bosque se dispone en esta capa superficial y la superficie media de las hojas de esta capa es notablemente menor que las de las hojas de los estratos inferiores (fig. 2) lo que de acuerdo con HORN (1971) y GIVNISH (1979) se interpreta como una adaptación a condiciones de alta iluminación. Puesto que la intensidad de

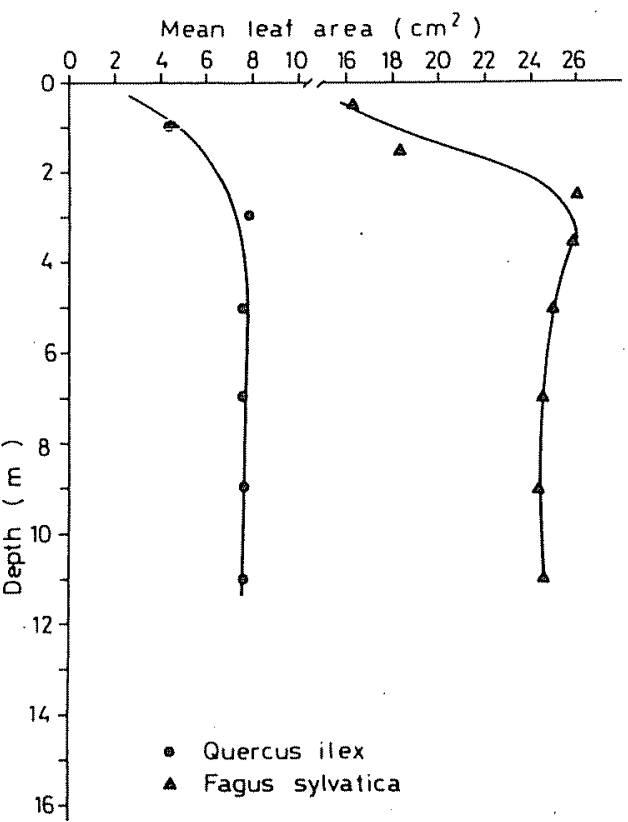

Figura 2. Distribución vertical de la superficie media de las hojas de haya y encina en el Montseny (Barcelona).

la radiación es elevada, la cantidad de clorofila presente en las hojas de estc nivel debe ser independiente de la misma, ya que se encuentra en exceso. A partir. del nivel en el que la radiación ha sido atenuada hasta el punto de que los fotosistemas dejan de estar saturados, la cantidad de clorofila presente en la hoja se relaciona más estrechamente. con la intensidad de la radiación fotosintéticamente activa incidente (PAR fig. 3). El resultado es que, si integramos la cantidad total de clorofila presente en una columna del bosque, se produce una distribución vertical con un máximo subsuperficial semejante a la distribución caracteristica de los ecosistemas acuáticos (fig. 1).

El hecho de que las hojas del nivel superior sean de menor tamaño es otra manifestación de las estrechas adaptaciones del bosque para captar la luz. Las hojas superficiales, de pequeño tamaño, tienen sentido sólo en la parte más elevada donde la tasa de fotones incidentes es alta y los centros 


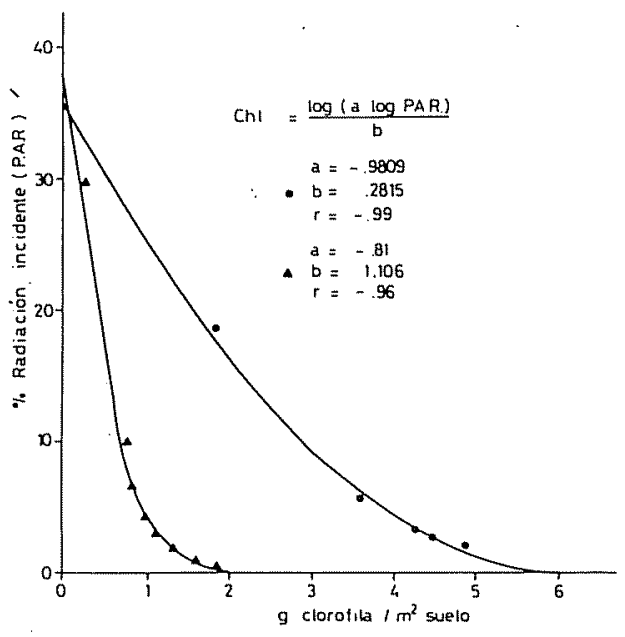

lïgura 3. Relación entre la cantidad de clorofila a $i^{-}$b y el porcentaje de la radiación incidente que alcanza a cada punto del perfil vertical de un encinar y un hayedo en el Montseny. La relación deja de ser valida para porcentajes supcriores al 36 y 39 por ciento respectivamente, que corresponde al nivel de saturación de los fotosistemas.

activos de los fotosistemas tienen gran probabilidad de ser alcanzados a la vez que posibilitan la exposición de mayor superficie foliar a la luz intensa sin producir una excesiva atenuación de la misma. A medida que descendemos en la copa y la radiación se hace menos intensa, no tiene sentido mantener tanta cantidad de clorofila (una molécula metabólicamente cara) en las hojas ya que la probabilidad de que sus centros activos sean alcanzados por los fotones, cada vez menos frecuentes, disminuye. Es más interesante disponer superficies laminares mayores, o lo que es lo mismo, hojas más grandes, para asegurarse la posibilidad de interceptar la luz. Importa más extender la clorofila presente que aumentar la cantidad de la misma. Es la misma estrategia que podemos observar en comunidades acuáticas y que viene representada por el caso de las grandes Lalminaria en un extremo ya que disponen toda su clorofila en un plano paralelo a la superficie $y$ en el otro extremo por las pequeñas cloroficeas fitoplanctónicas que llegan a mantener mayores cantidades de clorofila en una colum na de agua, permitiendo una mayor penetración de la luz.

$Y$ aún hay más: en cualquier ecosistema se manifiesta una tendencia en el sentido de aumentar la producción por unidad de energía utilizada lo cual, es otra manifestación del retardo en el empleo de la energía. En los ecosistemas acuáticos la capa superficial de agua es la más productiva. También es la qije recibe y degrada mayor cantidad de energía porque además de la luz que se absorbe en los primeros metros, los vientos y el calor ponen en juego mecanismos que resultan innprescindibles para la circulación de nutrientes y para la vida. HUTCHINSON (1941) al modelizar el metabolismo intermediario de los lagos estratificados encuentra que esta primera capa, que se corresponde de cerca con el epilimnion, sigue pautas de funcionamiento diferentes de las que siguen las capas inferiores debido al exceso de energia que éstas reciben en forma de materia órganica.

En un ecosistema forestal no puede hablarse de turbulencia en el mismo sentido con que lo hacemos en un embalse $o$ en un lago, dada la presencia de estructuras rigidas. Bien es verdad que existen diferencias importantes en la difusión de calor o de gases como el $\mathrm{CO}_{2}$ en condiciones de calma y con fuerte viento, pero las hojas de los árboles tienen una escasa capacidad de movimiento y ocupan posiciones casi permanentes si se las compara con las células del fitoplancton. Alhora bien, existen otros mecanismos de degradación de la energía en la capa superior de la bóveda forestal. La transpiración más elevada y el calentamiento de las hojas en esta zona superior son dos de ellos y por ello también a este nivel la producción es más elevada (al igual que sucedía en el lago) porque el bosque dispont la mitad de su superficie foliar y má: de la mitad de la clorofila en esta capa (figs. 1 y 2 ). 
GIVNISH, T. 1979. On the adaptative significance of leaf form in: Topics in plant population biology, SOLBRIG et. al. (eds.). Columbia University Press. págs: 375-407.

GRACIA, C. en prensa. Vertical structure of the forest: mechanisms of light interception and chlorophyll content in leaves. Forest Science.

HORN, H.S. 1971. The adaptative geometry of trees. Princeton Univ. Press. 144 págs.

HUTCHINSON, G.E. 1941. The mechanisms of intermediary metabolism in stratified lakes.
Ecological monographs, 11 (1): 23-60.

LIETH, H. \& WHITTAKER, R.H. (eds.). 1975. Prinary productivity of the biosphere. Springer Verlag, New' York. 339 pags.

MARGALEF, R. 1975. Diversity, stability and maturity in naturel ecosystems. In: Unifying concepts in Ecology. VAN DOBBEN \& LOVEMC CONELL (eds.). Dr. W. Junk The Hague. págs. 151-160.

1981. La Biosfera entre la termodinámica y el juego. Omega: Barcelona. 236 págs. 